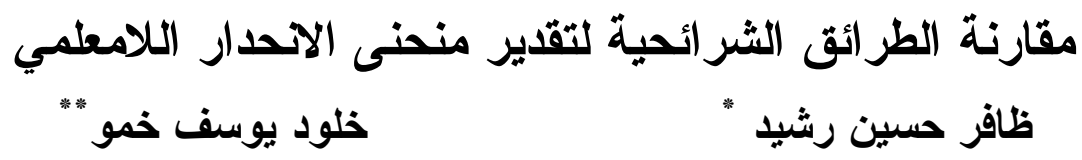

$$
\begin{aligned}
& \text { الملخـــص } \\
& \text { يهذف هذا البحث الى تتاول طر ائق متعلقة بتمهيد دوال الانحدار اللامعلمي } \\
& \text { بهدف ايجاد افضل الطر ائق التي تلائم نماذج منتوعة. و الى بحث ما قدمته البحوث } \\
& \text { الحديثة في مجال الانحدار اللامعلمي بهدف إيجاد طر ائق بديلة أو محورة نكون } \\
& \text { كفؤءة في معالجة حالات الإخفاق في الطر ائق المتناولة مثنلا لمعالجة حالة الاخفاق } \\
& \text { في طريقة تقليص الجزاء التي تعتمد على نموذج شريحة الانحدار متعددة الحدود } \\
& \text { المبتورة لما يتعلق بالنماذج الخاصة تطلبت الحاجة الى اقتر اح تحوير للعقد يجعل } \\
& \text { الطريقة اكثر كفاءة. وقد تمت صياغة نموذج محاكاة ولتوزيعات مختلفة للخطأ } \\
& \text { العثوائي ولنماذج ومستويات نباين مختلفة. كما تمت صياغة نموذج محاكاة } \\
& \text { ولنماذج مختلفة، وللتحقق من أداء هذه الطر ائق تم استخدام عدة مقاييس. }
\end{aligned}
$$

\title{
Comparison of Spline Methods for estimating Nonparametric
} Regression Curve

\section{ABSTRACT}

This research is focusing on methods related to smoothing Nonparametric Regression Functions. This is for the purpose of producing the best methods convenient for various methods. Thus, the most important purpose of the research is to find what the studies so far have offered in the field of Nonparametric Regression. Also to find alternative or modified methods, which are reliable for the treatment of failure regarding the methods in use, for example for the treatment of failure for penalized shrinkage method which depends on truncated Polynomial Regression spline model, especially for special models ,we suggest modification of knots make method more efficiency.

" ماستاذ/كلية الادارة والاقتصاد/ جامعة بغداد

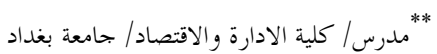

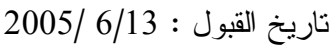

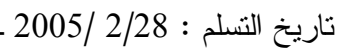


A simulation model has been performed with different distributions, for a number of methods. To verify the performance of such methods, many criteria have been carried out.

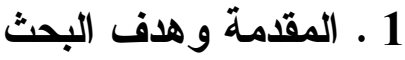

أن منحنى الانحدار يصف العلاقة العامة بين المتغير التوضيحي X ومتغير

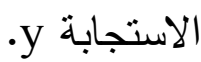

$$
\mathbf{y}_{\mathbf{i}}=\mathbf{g}\left(\mathbf{x}_{\mathbf{i}}\right)+\epsilon_{\mathbf{i}} \quad \mathbf{i}=1, \ldots, \mathbf{n}
$$

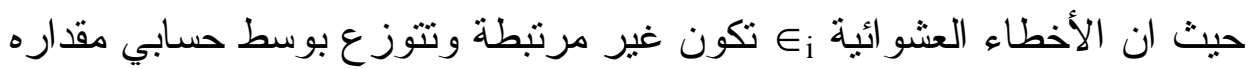

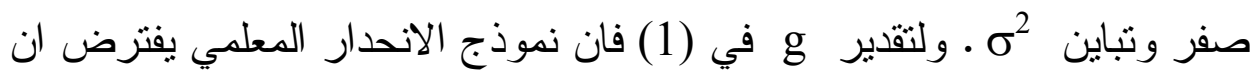

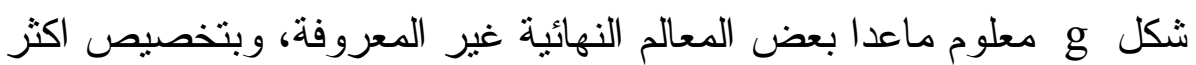
يفترض هناك متجــــــا من المعالم

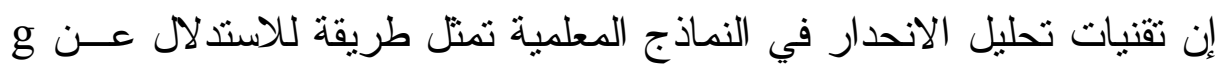

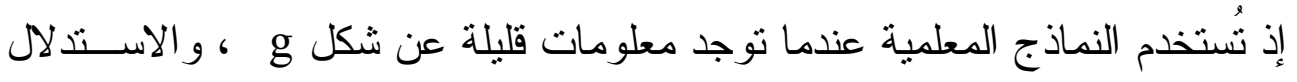

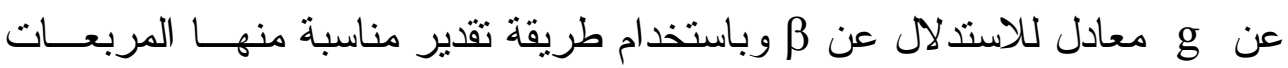
الصغرى الاعتيادية OLS أو الامكان الاعظم ML و أو أسلوب بيز في حالة نوفر

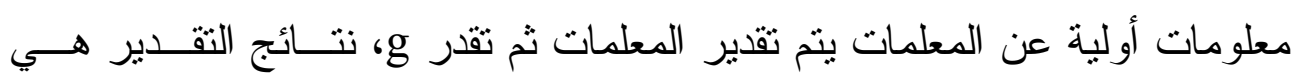

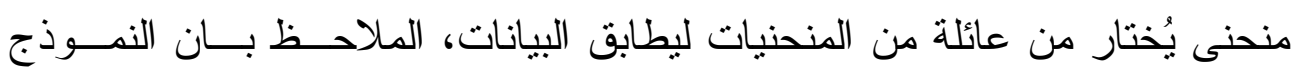

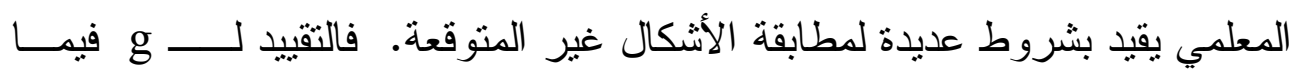

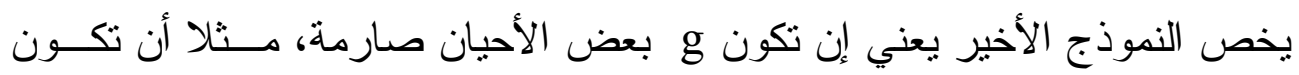

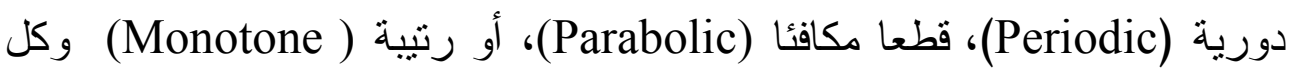

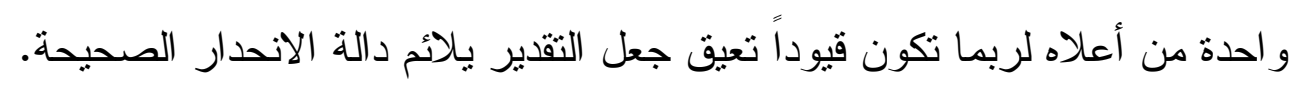

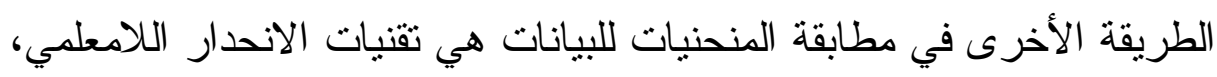

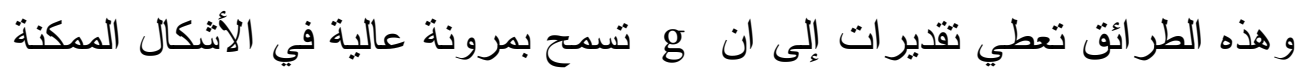

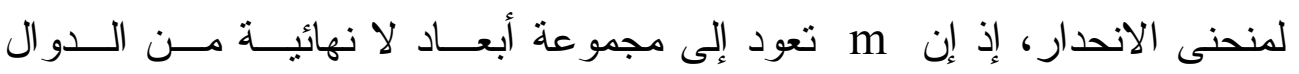

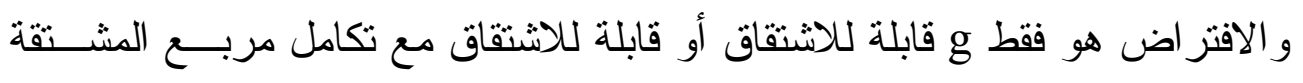

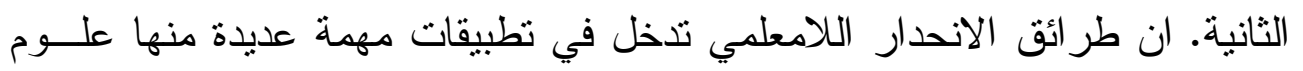




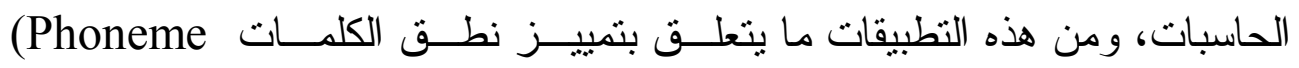
Recognition)

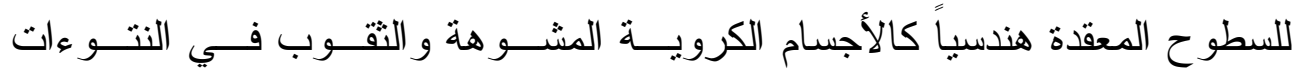

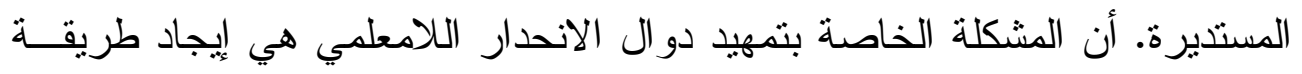

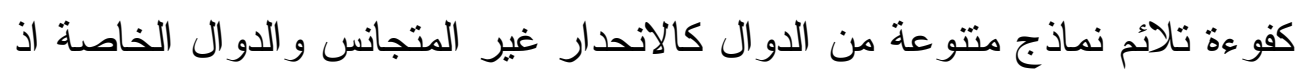

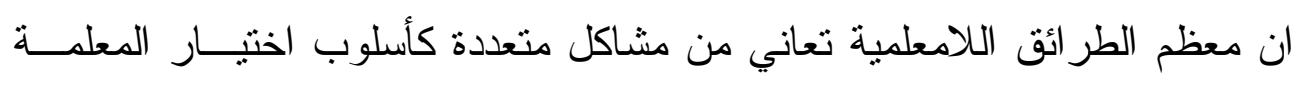

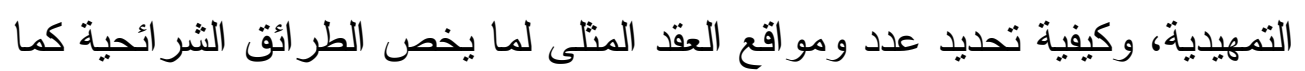

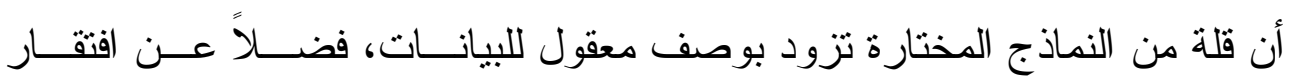

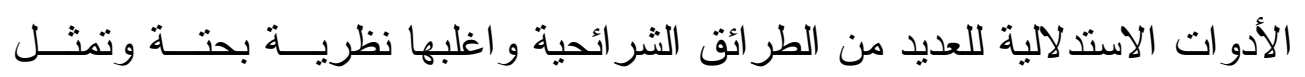

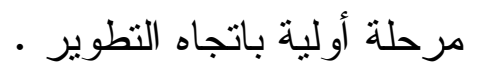

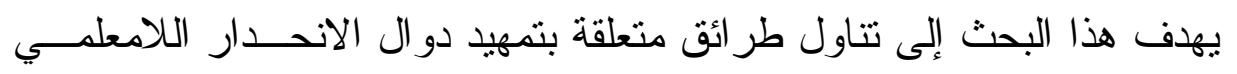

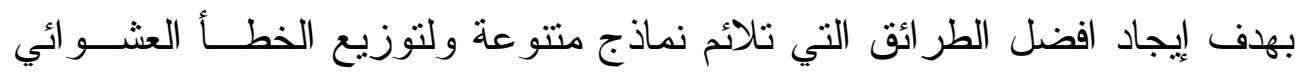
بحالته الطبيعية ـ كما يهدف الى بحث ما قدمته البحوث الحديثة في مجال الانحدار

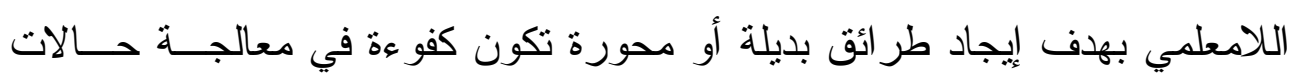

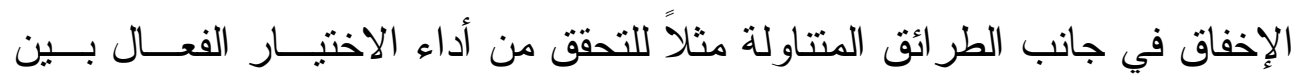

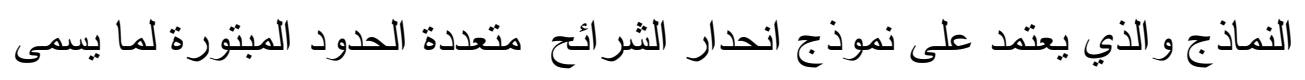

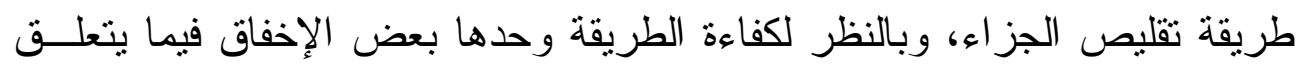

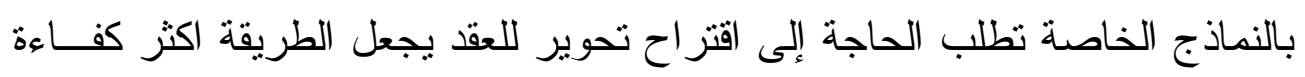

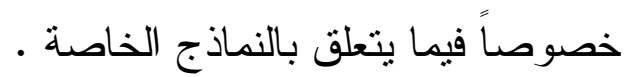

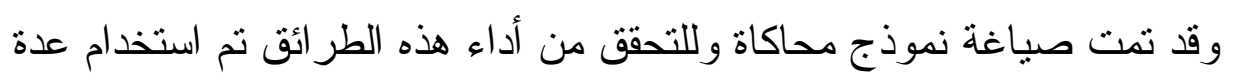

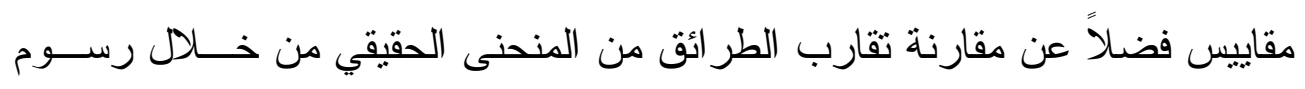
التي تعتمد على نتائج مستخلصة من تجارب المحاكاة. 


\section{2-1 الطرائق الثرائحيــــــة}

يمكن تعريف النماذج الثر ائحية (Spline Models) [5] بأنها نماذج المتغير

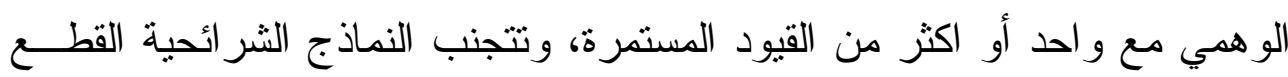

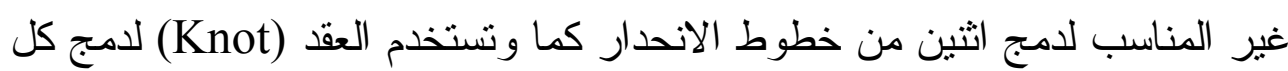

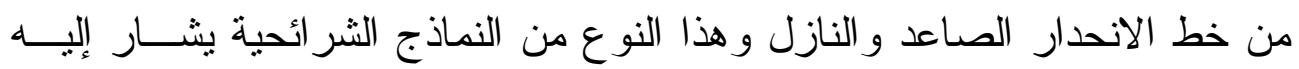
بنموذج الانحدار الخطي القطعي (Piecewise Linear Regression Model)

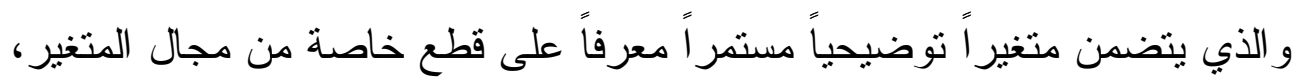

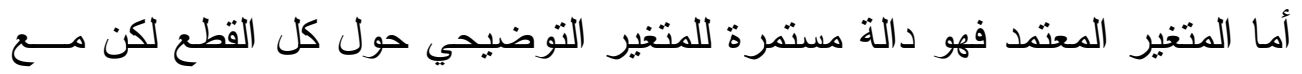
ميل مختلف لكل جز ء منفصل.

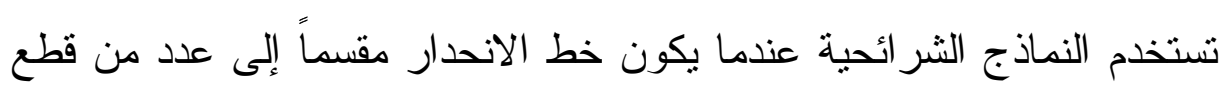

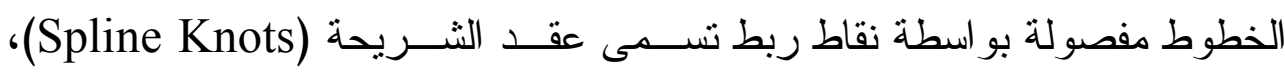
ويغير خط الانحدار اتجاهه عند نقاط الربط هذه، لكنه لا يقفز عند هذه النقاط. 1-1-2 الطريقة الثر ائحية مع عقد مكيفة يمكن تقدير g في (1-1-1) باستخدام أدناه.

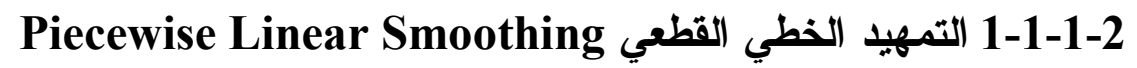

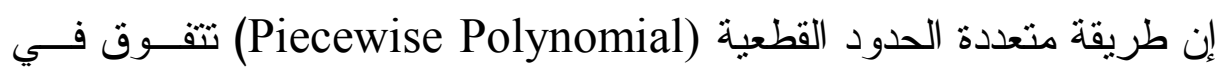
تحقيق مرونة اكبر لكن على حساب التمهيد الموضعي فالدالة العمومية (Global)

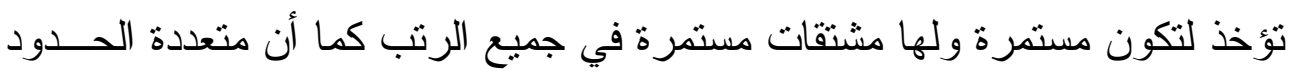

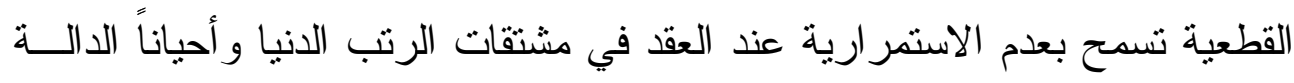

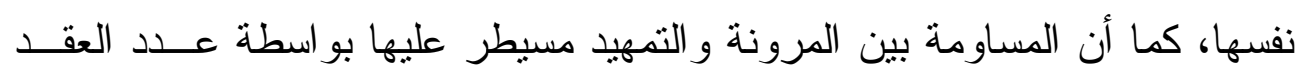

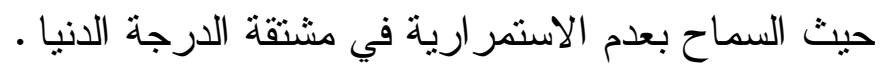
لتقدير g المعطاة في (1) فان : بطان

$$
\hat{\mathbf{g}}(\mathbf{x})=\mathbf{a}_{0}+\mathbf{a}_{1} \mathbf{x}+\sum_{\mathbf{k}=1}^{\mathrm{K}} \mathbf{a}_{\mathbf{k}} \mathbf{b}_{\mathbf{k}}(\mathbf{x})
$$


ولعدد ثابت من العقد K اذ الهذف منوضع العقد بحيث نحصل على اقـلـل قيهــة

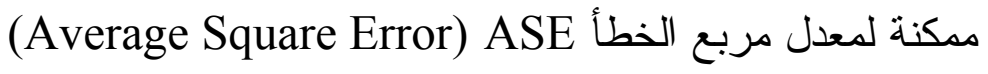

$$
\operatorname{ASE}=(1 / \mathrm{n}) \sum_{\mathrm{i}=1}^{\mathrm{n}}\left[\mathrm{y}_{\mathrm{i}}-\hat{\mathrm{g}}\left(\mathrm{x}_{\mathrm{i}}\right)\right]^{2}
$$

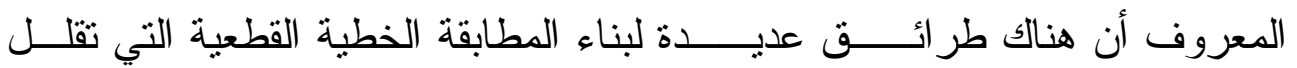

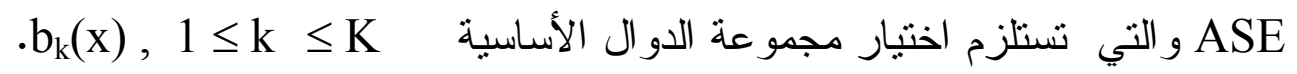

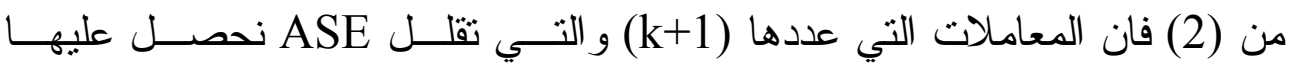

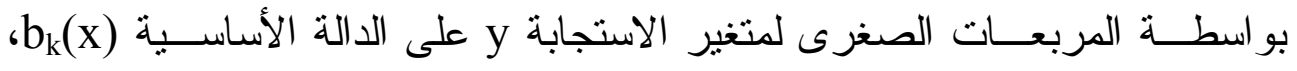

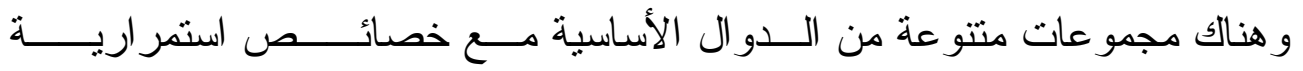

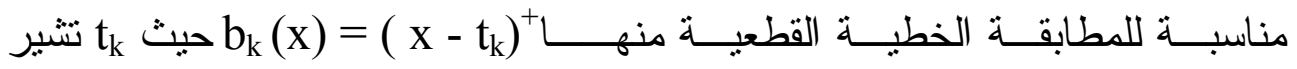

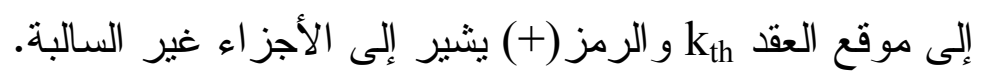

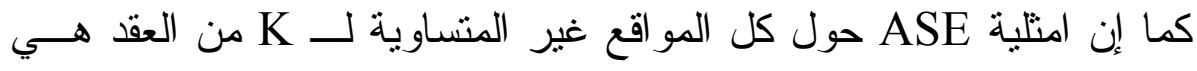

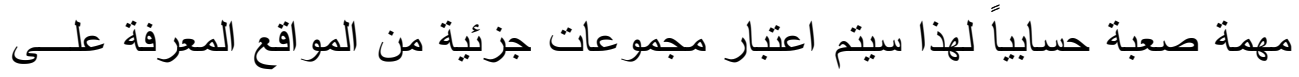

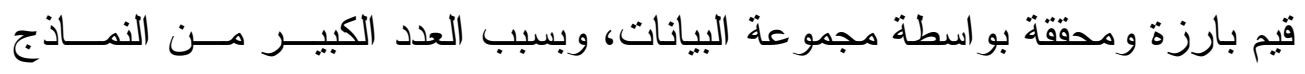

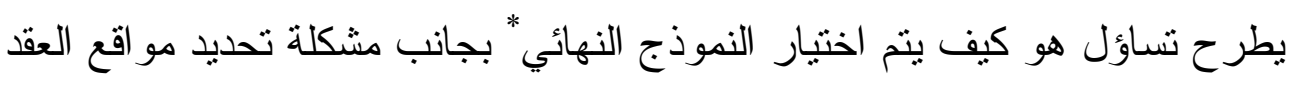

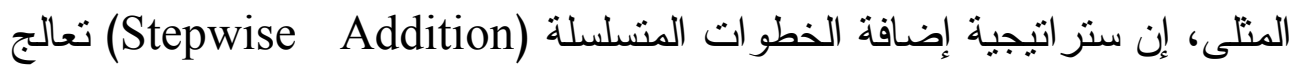

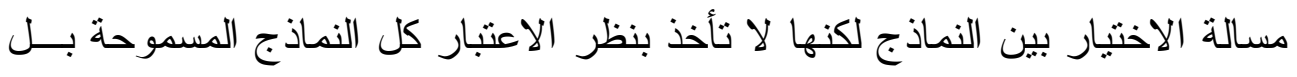

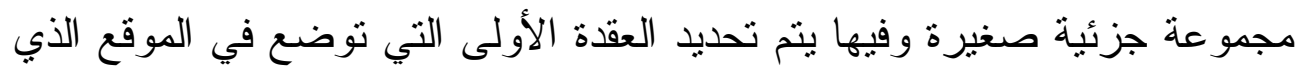

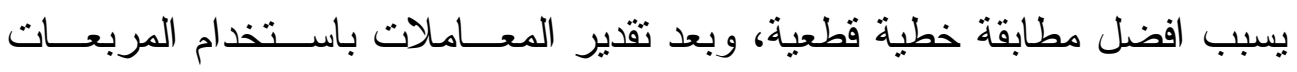

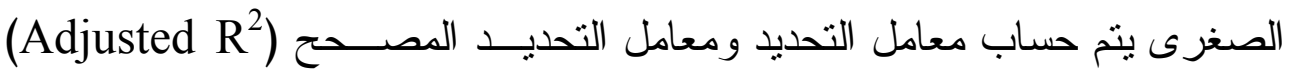

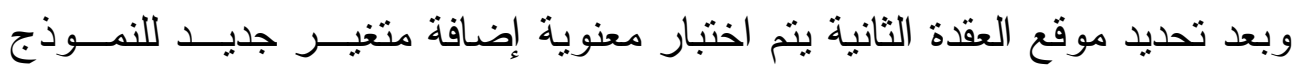

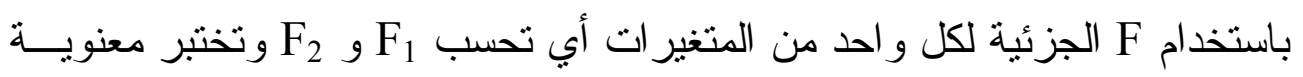

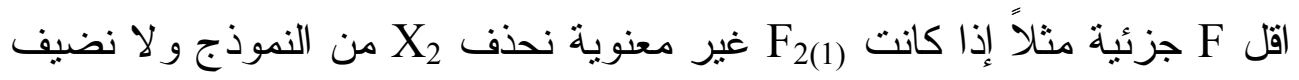
متغير جديد (عقدة جديدة) كذلك يحسب معامل التحديد R2 ومعامل التحديد المصحح

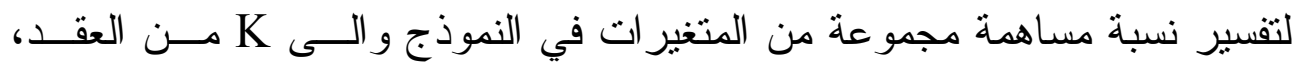
* الجزء النظري تم وضعه من قبل الباحئة كذلك أسلوب وضع العقد كوفا غير مطروقة في البحوث لكن الطريقة ليست مقترحة. 
وبسبب وجود سلسلة من النماذج كل نموذج فيه عقدة إضــافية مقارنــة بالســابق

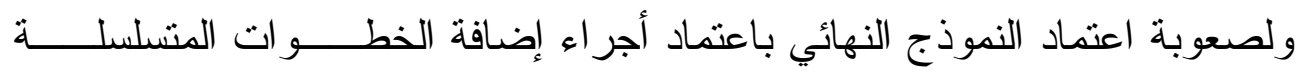

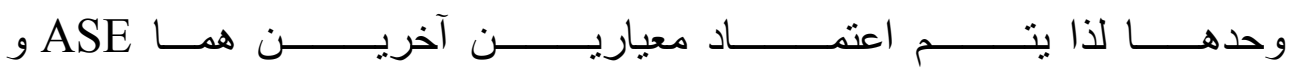
(Generalized Cross Validation) GCV

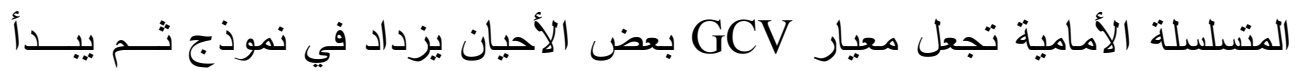

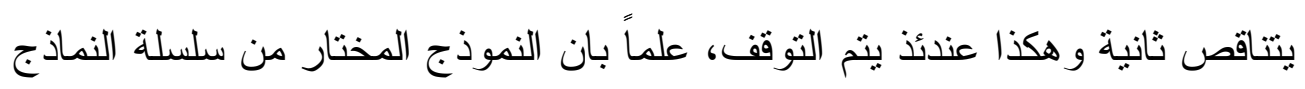
هو الذي يقلل معيار GCV حيث:

$$
\begin{aligned}
\mathbf{G C V}=(1 / \mathbf{n}) \sum_{i=1}^{n}\left[\mathbf{y}_{\mathbf{i}}-\hat{\mathbf{g}}_{-\mathbf{i}}\left(\mathbf{x}_{\mathrm{i}}\right)\right]^{2} /[1-\mathbf{d}(\mathbf{K}) / \mathbf{n}]^{2} \quad \ldots(3) \\
\\
. \mathrm{d}(\mathrm{K})=3 \mathrm{~K}+1 \text { و }
\end{aligned}
$$

Piecewise Cubic Fitting ${ }^{[3]}$ 2-1-1-2 المطابقة التكعيبية القطعية

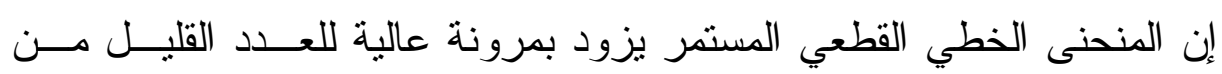

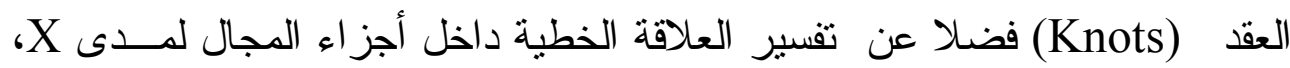
لكنه يخفق في تحقيق الاستمر ارية في المشتقة الأولى و الثانبة عند كل موقع للعقد.

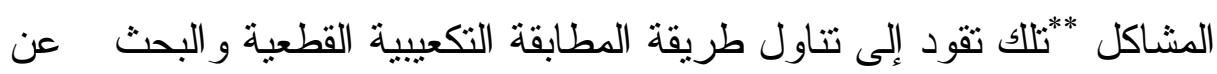

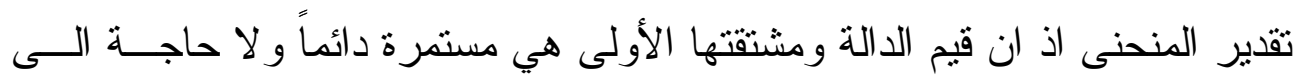
قيود إضافية وهي استمر ار المشتقة الثانية، إن تقدير المنحنى التكعيبي يكون: $\hat{\mathbf{g}}_{\mathbf{c}}(x)=\mathbf{a}_{0}+\mathbf{a}_{1} \mathbf{x}+\mathbf{a}_{2} \mathbf{x}^{2}+\mathbf{a}_{3} x^{3}+\sum_{k=1}^{K} \mathbf{a}_{k} B_{k}(x) \quad \ldots$ (4) أن اتجاه العقد

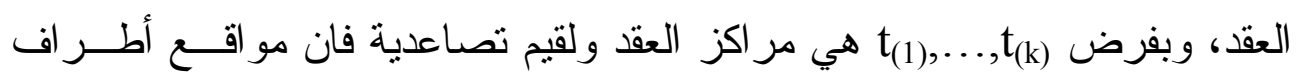

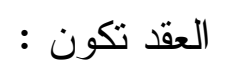
$\mathbf{t}_{(\mathrm{k})-}=\left(\mathbf{t}_{(\mathrm{k})}+\mathbf{t}_{(\mathrm{k}-1)}\right) / \mathbf{2}, \quad \mathbf{t}_{(\mathrm{k})+}=\left(\mathbf{t}_{(\mathrm{k})}+\mathbf{t}_{(\mathrm{k}+1)}\right) / 2 \quad 2 \leq \mathbf{k} \leq \mathrm{K}-\mathbf{1}$ إن تقدير المنحنى التكعيبي القطعي في (4) نحصل عليه بواسطة تقليـلـ مقيــاس نسبة للمعاملات ASE

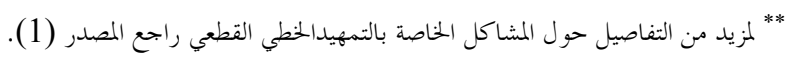




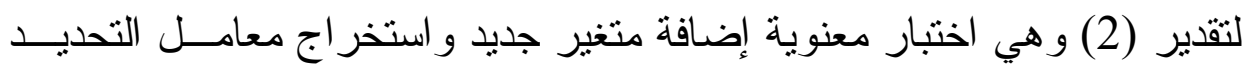

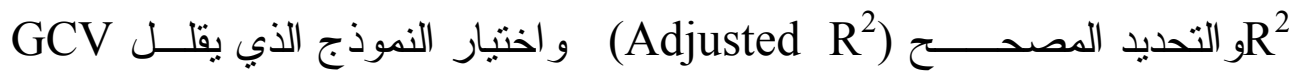
وذللك بهدف الوصول للنموذج النهائي.

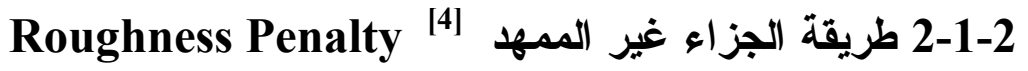

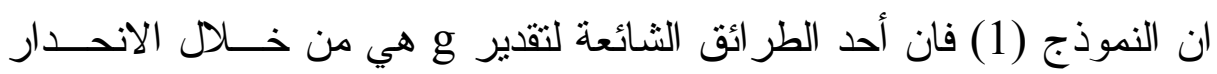

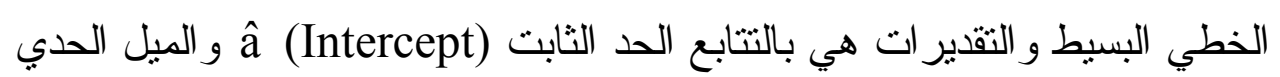

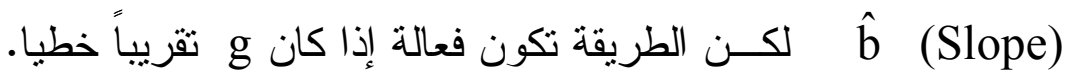

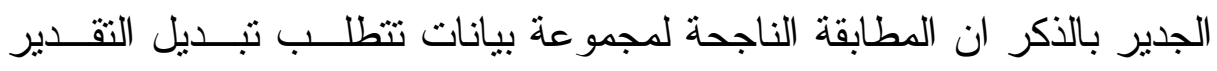

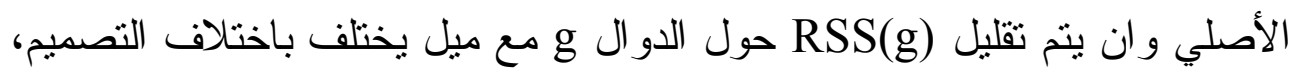
إن تقدير منحنى الانحدار بطريقة الجزاء غير الممهدة يكون بإعطاء أية دالة و قابلة

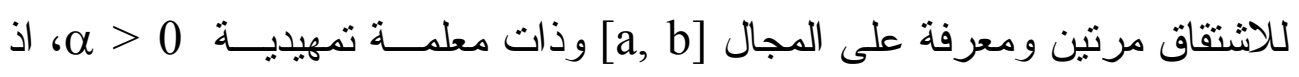
تعرف مجمو ع مربعات الجز اء كالآتي:

$$
S(g)=\sum_{i=1}^{n}\left[y_{i}-g\left(x_{i}\right)\right]^{2}+\alpha \int_{a}^{b}\left[g^{\prime \prime}(t)\right]^{2} d t \quad \ldots
$$

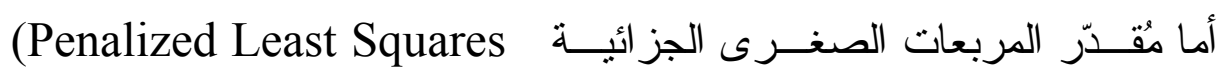
وفيعرف بأنه تقليل S(g) عstimator) مرتين، وان مصطلح الجز اء غير المههد

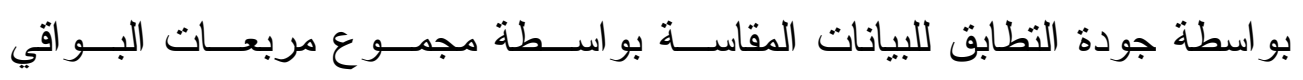

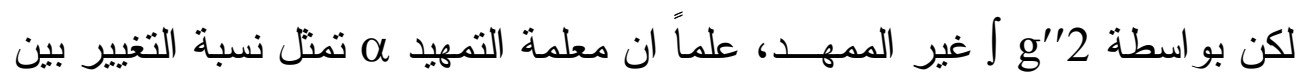
خطأ البو اقي و الاختلاف الموضعي، و الكمية تعطى في مصطلح مجموع مربعسات خطأ البو اقي وفقاً إلى وحدة تكامل مربع المشتقة الثنانية.

$\sum^{\mathrm{n}}\left[\mathrm{y}_{\mathrm{i}}-\mathrm{g}\left(\mathrm{x}_{\mathrm{i}}\right)\right]^{2}$

بفرض أن gâtural Cubic Spline) NCS هي شريحة تكعيبية طبيعية وبفرض ان المتجهات $\underline{\mathbf{y}}=\left(\mathbf{y}_{1} \ldots \mathbf{y}_{\mathbf{n}}\right)^{\prime} ; \underline{\mathbf{g}}=\left(\mathbf{g}_{1} \ldots \mathbf{g}_{\mathbf{n}}\right)^{\prime} ; \underline{\gamma}=\left(\gamma_{2} \ldots \gamma_{\mathbf{n}-1}\right)^{\prime}$ 


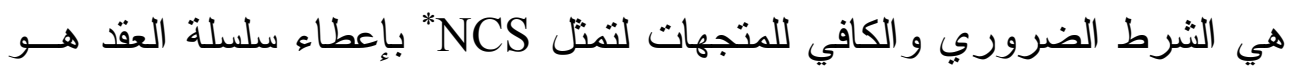
الاعتماد على مصفوفتي الحزمة R, Q و اللتين تعرفان كالآتي :

$$
\begin{aligned}
& \mathbf{q}_{j-1, j}=h^{-1}{ }_{j-1} ; q_{j j}=-h^{-1}{ }_{j-1}-h^{-1}{ }_{j} ; q_{j+1, j}=h^{-1}{ }_{j}, i=1, \ldots, n, j=2, \ldots, n-1 \\
& \mathbf{q}_{\mathrm{jj}}=\mathbf{0} \\
& \forall|\mathbf{i}-\mathbf{j}| \quad \geq 2
\end{aligned}
$$$$
r_{i i}=(1 / 3)\left(h_{i-1}+h_{i}\right) \quad, i=2, \ldots, n-1 ; r_{i, i+1}=r_{i+1, i}=(1 / 6) h_{i}, i=2, \ldots, n-2
$$$$
\mathbf{r}_{\mathrm{ij}}=\mathbf{0} \quad \forall|\mathbf{i}-\mathbf{j}| \geq \mathbf{2}
$$

حيث ان Q مصفوفة من درجة (n×(n-2)) للمتغير ات التوضيحية.

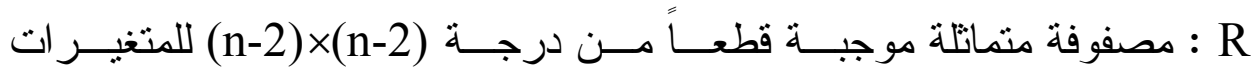

\section{التوضيحية.}

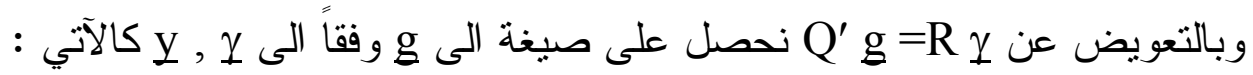

$$
\begin{aligned}
& \left|\mathbf{r}_{i i}\right|>\sum_{j \neq i}\left|\mathbf{r}_{i j}\right| \quad \forall \mathbf{i} \quad ; \mathbf{h}_{i}=\mathbf{x}_{i=1}-\mathbf{x}_{i} \quad, \quad \mathbf{i}=1, \ldots, \mathbf{n}-1 \\
& \mathrm{~g}=\mathrm{y}-\alpha \mathrm{Q} \gamma
\end{aligned}
$$

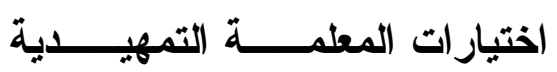

1. مقياس Cross Validation) CV)

ان الفكرة الأساسية للمقياس هي اختيار قيمة م التي تقلل CV(م) حيث ان

$$
\operatorname{CV}(\alpha)=n^{-1} \sum_{i=1}^{n}\left(y_{i}-\hat{g}^{(-i)}\left(x_{i} ; \alpha\right)\right)^{2}
$$

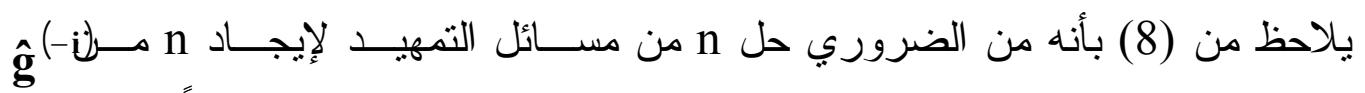

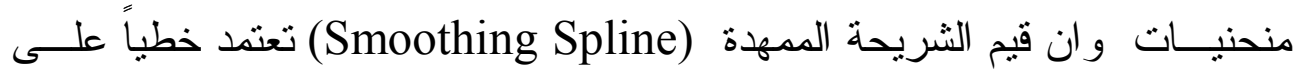
البيانات خل خل المعادلة y

$$
C V(\alpha)=n^{-1} \sum_{i=1}^{n}\left[\left(y_{i}-\hat{g}\left(x_{i}\right)\right) /\left(1-A_{i i}(\alpha)\right)\right]^{2}
$$


حيث أن البيانات الكاملة $\mathrm{A}_{\mathrm{ii}}(\alpha)$

(Generalized Cross-Validation) GCV مقياس 2

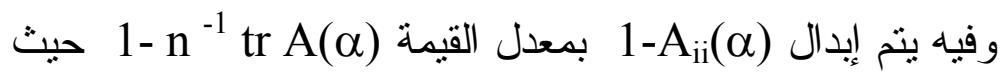
$\operatorname{GCV}(\alpha)=n^{-1} \sum_{i=1}^{n}\left[y_{i}-\hat{g}\left(x_{i}\right)\right]^{2} /\left[1-n^{-1} \operatorname{tr} A(\alpha)\right]^{2} \quad \ldots$ (10)

3-1-2 طريقة الجزاء غير الممهر مع اقتراح لاختيار المعلمة التمهيدية

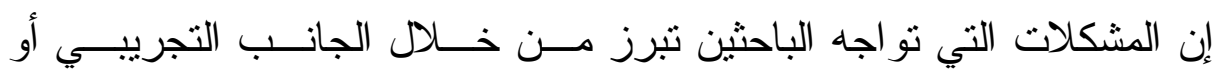

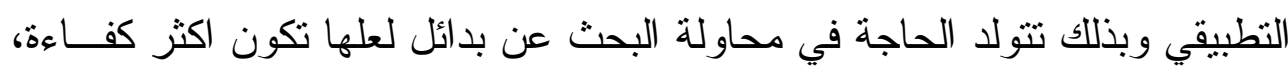

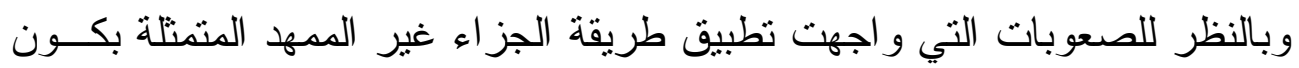

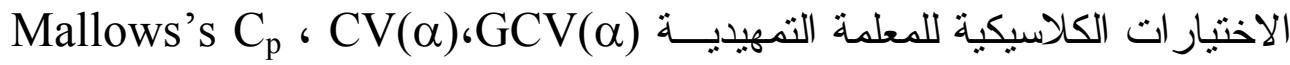

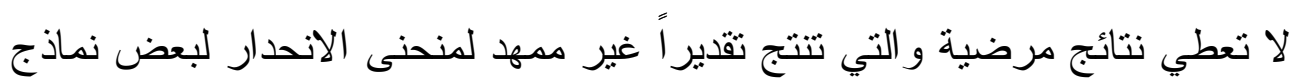

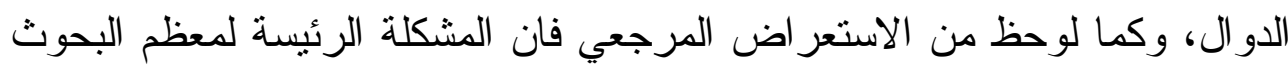

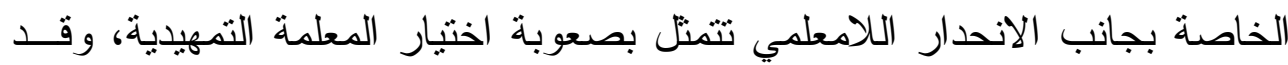

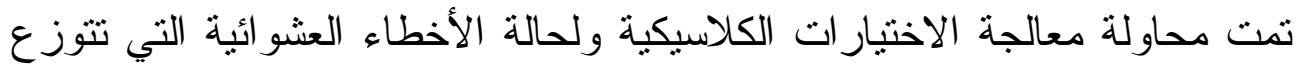

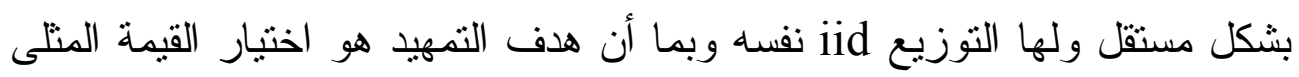

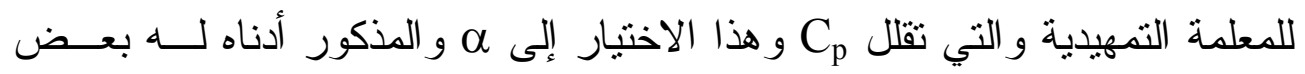
التقام مقارنة مع النوع الكلاسيكي ولني هو

$$
\hat{\boldsymbol{\sigma}}^{2}=\sum_{\mathrm{i}=1}^{\mathrm{n}}\left[\mathrm{y}_{\mathrm{i}}-\hat{\mathbf{g}}\left(\mathrm{x}_{\mathrm{i}} ; \boldsymbol{\alpha}\right)\right]^{2} /\left[\mathrm{n}-\operatorname{tr} 2 \mathrm{~S}\left(\boldsymbol{\alpha}^{*}\right)\right]
$$

وأن * هي القيمة الصغرى إلى $\alpha$ أما مصفوفة hat فهي

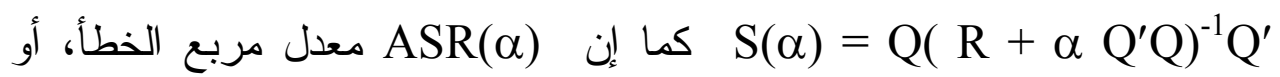
بو اسطة مقياس GCV(

$$
\begin{gathered}
\operatorname{Modified~C}_{\mathrm{p}}(\alpha)=\operatorname{ASR}(\alpha)+2 \operatorname{tr} S(\alpha) \hat{\sigma}^{2} / \mathbf{n} \\
\text { ModifiedGCV }(\alpha)=\operatorname{ASR}(\alpha) /[1-\operatorname{tr} S(\alpha) / n]^{2}
\end{gathered}
$$




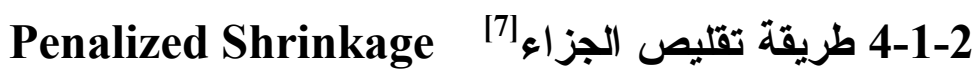

تعتمد الطريقة على استر اتيجية فعالة للاختيار بين كل النماذج الممكنة باعتمــاد

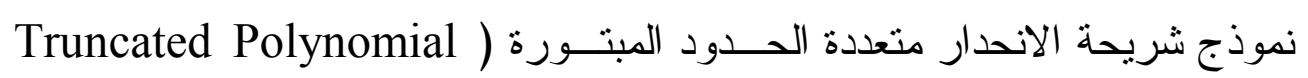
$y$ حيــ (Regression Spline Model متجه متغير الاستجابة، مصفوفة المتغير التوضيحي وان Xi أما ان تكون أعداد حقيقية أو عينة عشوائية لتوزيع أحادي المتغير ات، و v(x) دالة أحادية موجبة، و و متجه الاخطاء العشو ائية وله متوسط صفر وتباين و احد . ان تقدير m(x) يمكن أن يوضع بالثكل الاتي:

$$
\hat{\mathbf{m}}(\mathbf{x})=X\left(X^{\prime} X+\alpha D\right)^{-1} X^{\prime} \underline{y} \quad \ldots(\text { (13) }
$$

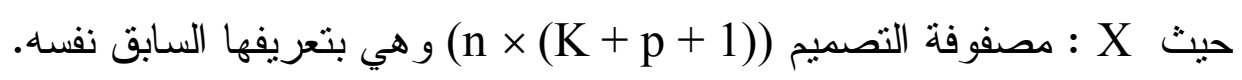

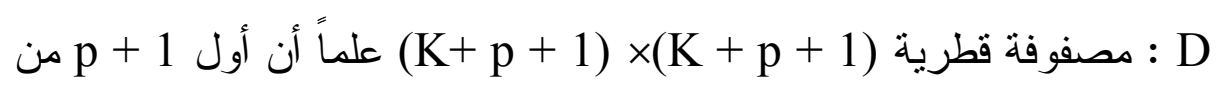

$$
\begin{aligned}
& X=\left[1, \mathbf{x}, \mathbf{x}^{2}, \ldots, \mathbf{x}^{\mathbf{p}},\left(\mathbf{x}-\kappa_{1}\right)_{+}^{\mathbf{p}}, \ldots,\left(\mathbf{x}-\kappa_{\mathbf{k}}\right)_{+}^{\mathbf{p}}\right] \\
& \text { عناصر ها أصفار و K من العناصر الباقية قيمها و احد. } \\
& \text { • p و القوة الأساسية المبنورة هي من الدرجة (u) }
\end{aligned}
$$

א : العقد (Knots لمتعددات الحدود القطعية التي تتتج من التوفيق الخطي للدالة الأساسية. إن زيادة درجة p ستؤدي إلى حالة التعدد الخطي لذا يتم بتر القوى وتســتخدم p = 3 اما اختيار العقد للنموذج فيكون وفقاً للقاعدة الاتية: $\kappa_{k}=\left(x_{(d k)}+x_{(d k+1)}\right) / 2 \quad k=1, \ldots, K$

وان [ ه وجود على الأقل d من المشاهدات بين كل عقدة، وفيما يلي مقاييس لاختيار ه: C 1 ورذا المقياس افترضه Mallows حيث : $C_{p}(\alpha)=\operatorname{RSS}(\alpha)+2 \hat{\sigma}^{2} \operatorname{tr}\left[X^{\prime} X\left(X^{\prime} X+\alpha D\right)^{-1}\right]$ 


$$
\begin{gathered}
\text { ان } \\
\hat{m}(\alpha) .
\end{gathered}
$$

(Generalized Cross-Validation) * GCV مقياس

ويأخذ هذا المقياس الثنكل :

$$
\operatorname{GCV}(\alpha)=\sum_{i=1}^{n}\left(y_{i}-\hat{g}\left(x_{i}\right)\right)^{2} /(n-\operatorname{tr}(H))^{2} \quad \ldots \text { (15) }
$$

وان H = X [ X'X

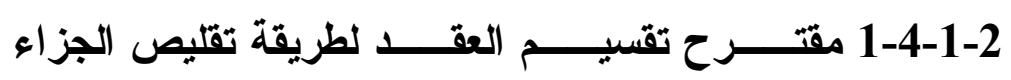

من خلال استعر اض معظم البحوث الخاصة بشريحة الانحـــار لـــوحظ أن

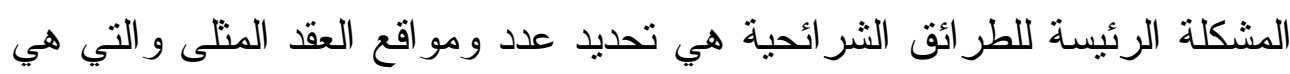

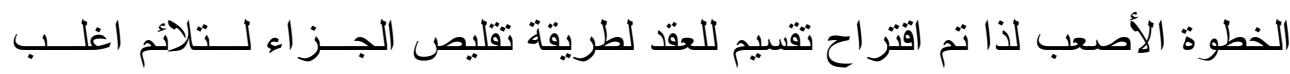

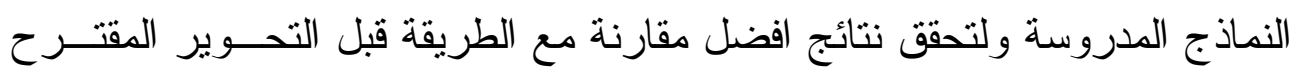

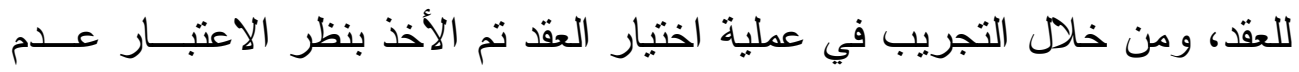

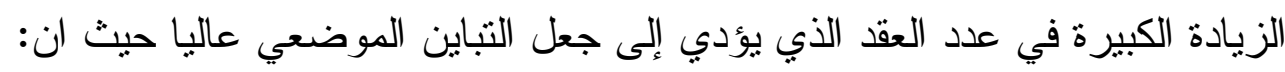

$$
\begin{aligned}
K & =\max [n / 3.5,10], d=\min [3,(n / 10)] \\
\kappa_{k} & =\left(x_{(d k)}+x_{(d k+1)}\right) / 2
\end{aligned}
$$

علماً أن هذا التخصيص يؤكد بأنه على الأقل يوجد d من المشاهدات بين كل

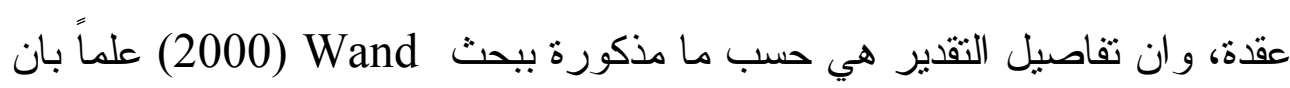
أفضلية التقسيمات للعقد تمت من خلامل التجريب في جانب المحاكاة.

5-1-2 الخطوات المتسلسلة Stepwise POLYMARS [ 2، 6.5 ] لاختيار

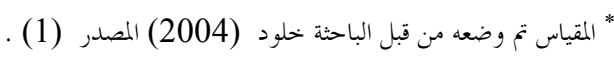


لتوضيح خطوات خوارزمية شرائح الانحدار المكيفة المتعدة الأبعاد المتعددة

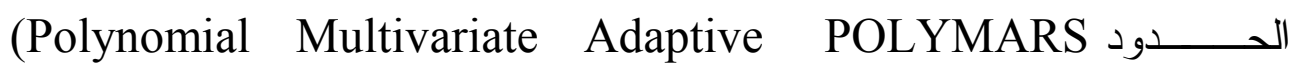
Regression Splines)

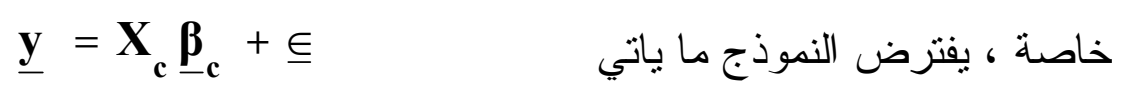
حيث: ل متجه (n×1) لمتغير الاستجابة.

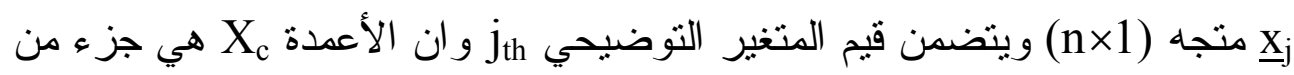
$\cdot \mathrm{x}_{\mathrm{j}}$ متجه

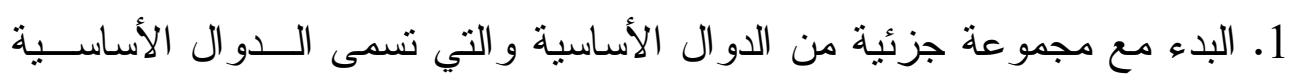

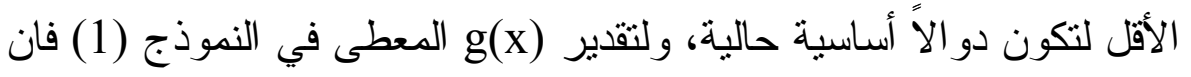
$\hat{g}(x)=a_{0}+a_{1} x+a_{2} x^{2}+\ldots+a_{q} x^{q}+\sum_{m=1}^{M} \beta_{m} B_{m j}(x) \quad \ldots$ (16) $\mathbf{B}_{\mathrm{mj}}(\mathbf{x})=\left(\mathbf{x}_{\mathrm{m}}-\widetilde{\mathbf{x}}_{\mathrm{mj}}\right)_{+}, \mathbf{1} \leq \mathbf{j} \leq \mathbf{J}_{\mathrm{m}}$ $X=\left[\begin{array}{llllll}1 & x & x^{2} & x^{3} B_{1}(x) \ldots B_{m}(x)\end{array}\right], \underline{\beta}=\left[\begin{array}{lllllll}a_{0} & a_{1} & a_{2} & a_{3} & \beta_{1} & \ldots & \beta_{m}\end{array}\right]^{\prime}$

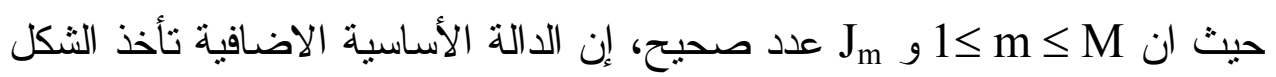

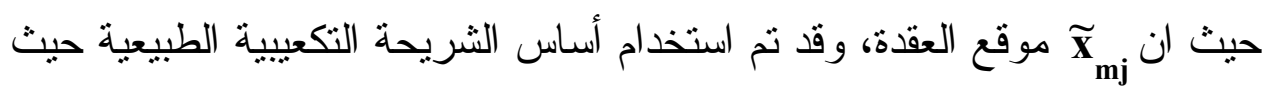
وان

2. تعاد الخطو ات حتى تصبح الدو ال الأساسية الحالية دو الاً أساسية كاملة.

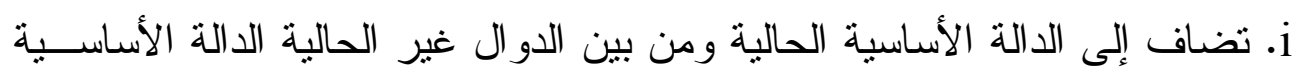
التي لها اكبر قيمة مطلقة لاحصاءة Rao.

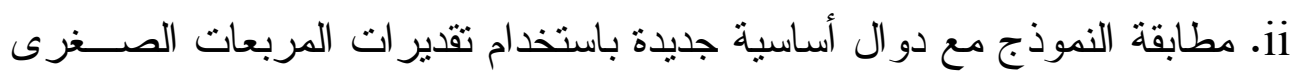
ومقياس GCV للمطابقة. 
3. حذف الخطوات المنسلسلة Stepwise Deletion تعاد الخطو ات حتى تصبح الدو ال الأساسية الحالية دو الاً أساسية اقل.

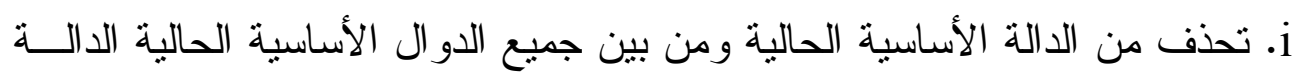
التي لها اقل قيمة مطلقة لاحصاءة Wald.

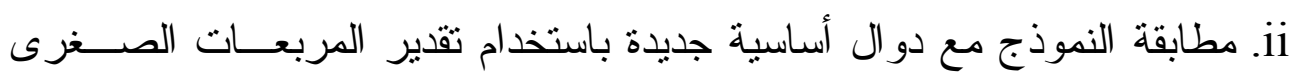
ومقياس GCV للمطابقة.

يكون للتقدير النهائي اقل GCV من بين كل النماذج المنطابقة في العمليات أعلاه.

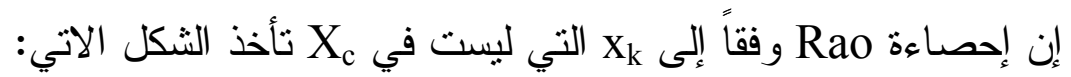

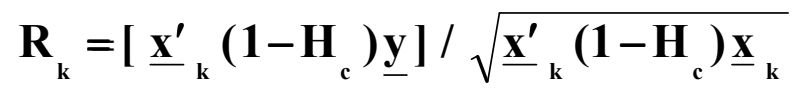

حصفوفة Hat $\mathrm{H}_{\mathrm{c}}=\mathrm{X}_{\mathrm{c}}\left(\mathrm{X}_{\mathrm{c}} \mathrm{X}_{\mathrm{c}}\right)^{-1} \mathrm{X}_{\mathrm{c}}^{\prime}$ وفقاً للنموذج الحالي أما احصــاءة

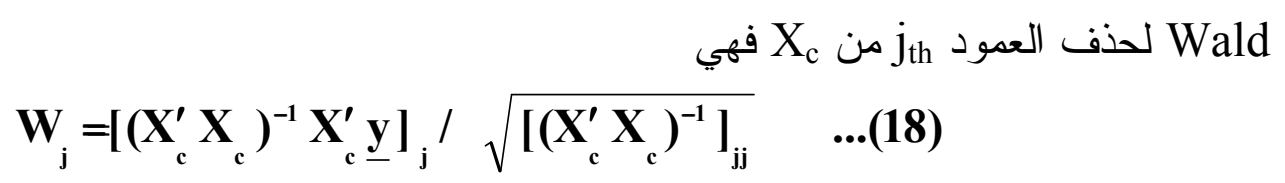

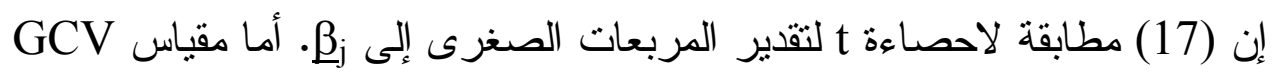
فيعطى بالثكل

$$
\text { GCV }=\mathbf{n}^{-1} \operatorname{RSS}_{J} /[1-\mathbf{a}(J-1) / \mathbf{n}]^{2} \quad \ldots \text { (19) }
$$

حيث RSS مجموع مربعات البواقي، J عدد الحدود في النموذج، a المعلمــة

$$
\begin{aligned}
& \text { وتؤخذ مساوية إلى 2.5. } \\
& \text { 3. الجاتب التجريبي }
\end{aligned}
$$

يتم اللجوء الى تجارب المحاكاة في الحالات التي يصعب الحصول فيها على ولى ولى

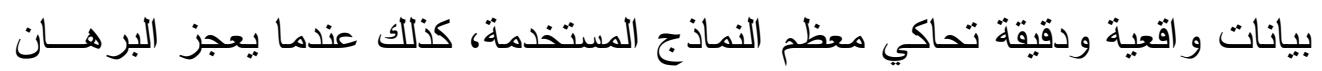

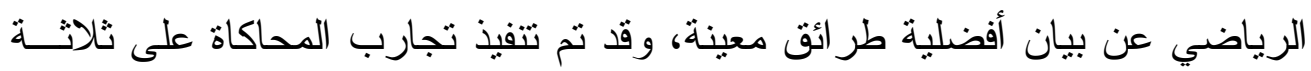

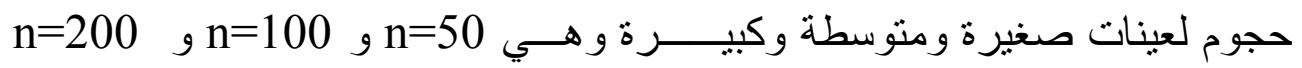

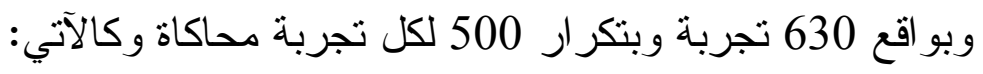




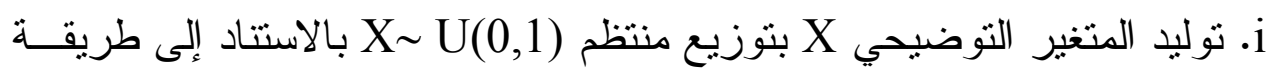

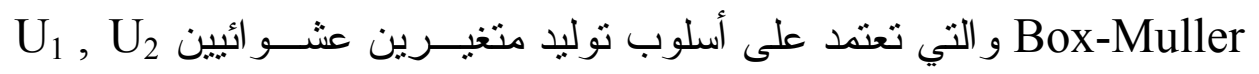
يتبعان التوزيع المنتظم

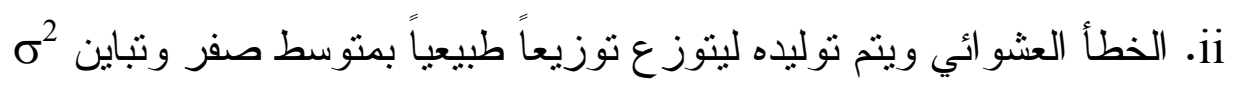
أب

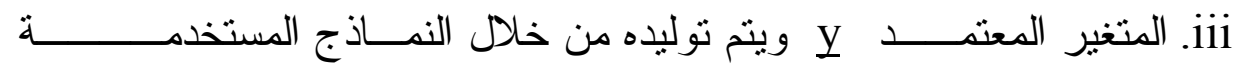

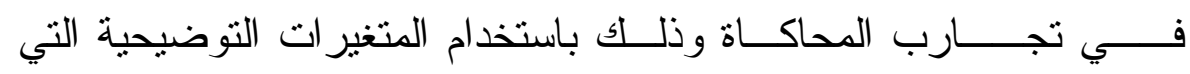

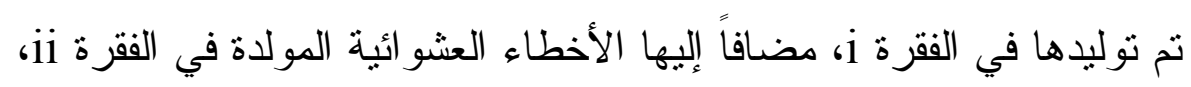
ولكل نموذج من النماذج المدروســــــة. 1-3 النماذج المستخدمة في تجارب المحاكاة

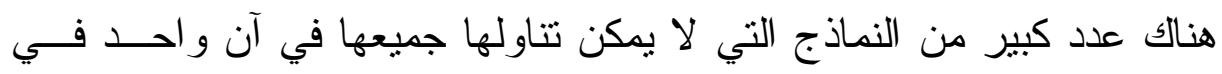

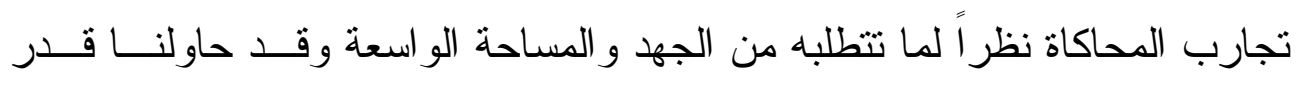

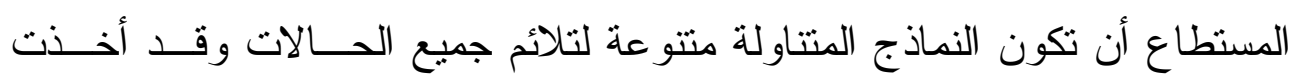
النماذج من بحوث منشورة كالآتي: 1 2. النموذج الخاص (تغير حيزي و اطئ في التذبذب) وصيغته هي (2x) $y=\sqrt{x(1-x)} \sin \left(\frac{2 \pi\left(1+2^{(9-4 j) / 5}\right)}{x+2^{(9-4 j) / 5}}\right) \quad j=3$

3. النموذج الخاص بدالة الانحدارغير المتجانسة وصيغته y=exp $\left(-400(x-0.6)^{2}\right)+(5 / 3) \exp \left(-500(x-0.75)^{2}\right)+2 \exp \left(-500(x-0.9)^{2}\right)$

2-3 تنفيذ تجارب المحاكاة لطرائق الأحدار اللامعلمي

لكل نموذج من النماذج المدروسة نم عمل ما بأني :

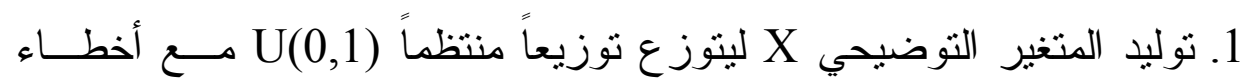
عشو ائية تتبع توزيعاً طبيعياً .

2. بالنسبة الى طر ائق الثر ائحية تم اعتماد عدة معايير لاختيار معلمة التمهيـــ

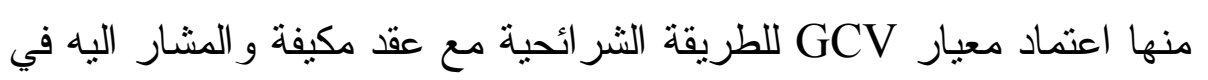




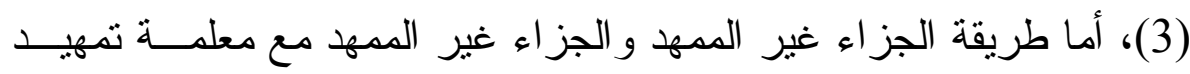

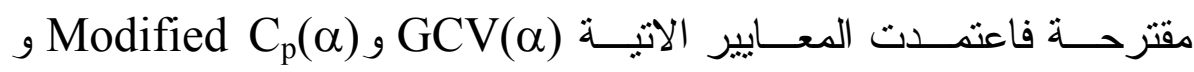

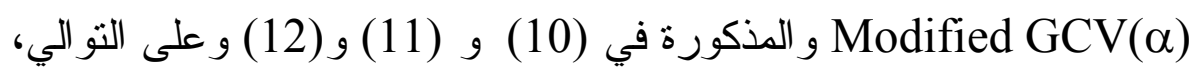

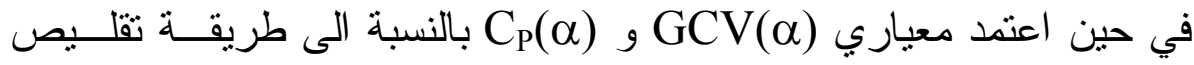

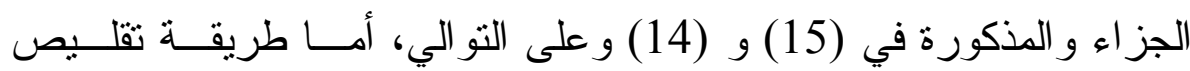

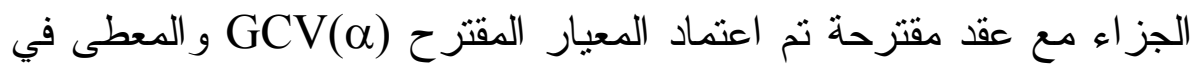

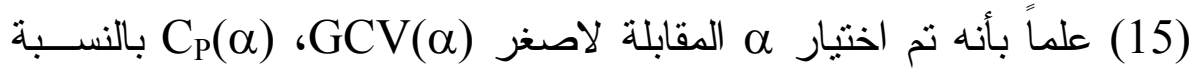

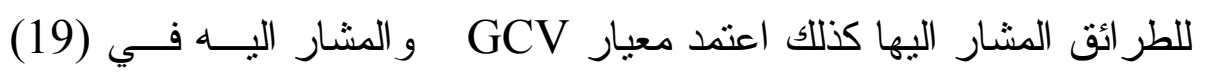
بالنسبة الى طريقة Stepwise POLYMARS.

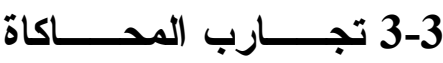
تم تحليل نتائج المحاكاة لكل نموذج وقد نم تفسير اغلب النماذج وكنلك الرسوم

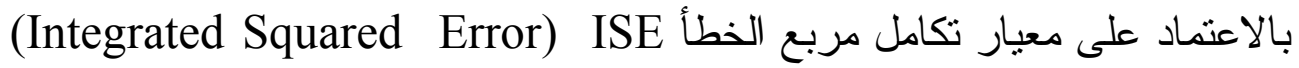
الذي تعتمده اغلب البحوث كونه يأخذ ما بين النقاط عند الثقدير وكما ياتي: النموذج الأول يلاحظ أن طريقة تقليص الجز اء للنموذج غير الخطي باعتماد التقسيم المقترح للعقد

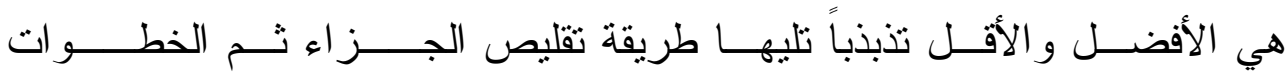

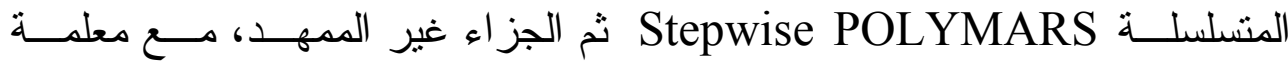

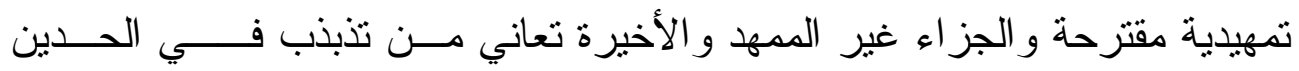

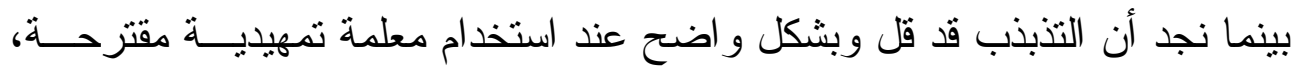

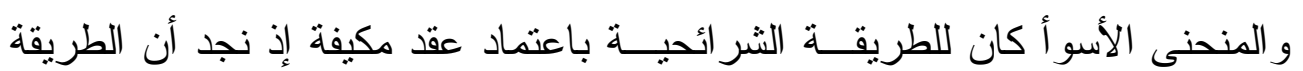

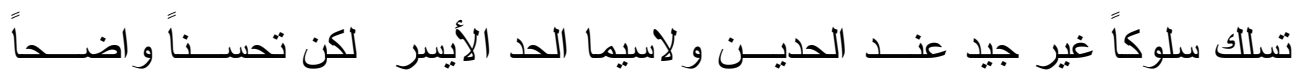

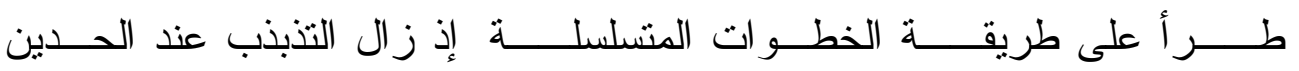
وكانت قريبة من النموذج غير الخطي ويمـكن ملاحظة ذلك في الثكلين (2) و (3) النموذج الثاني

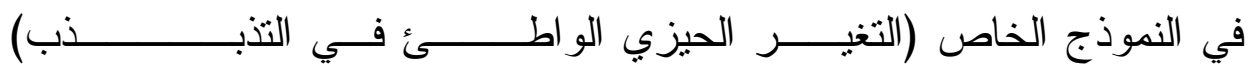
(Low Spatial Variability of Oscillation ) 


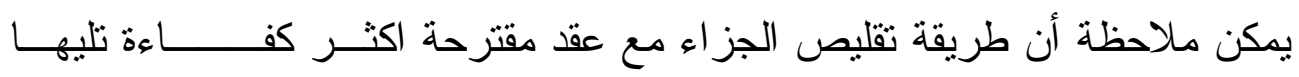
طريقة الجزاء غير الممهد مع معلمة تمهيدية مقترحة ثم تقليص الجز اء و الخطوات

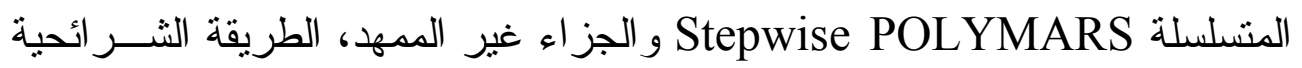

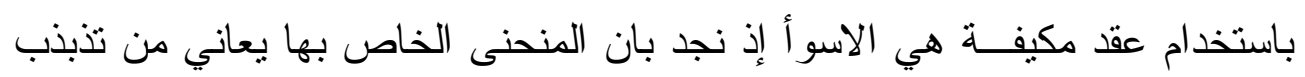

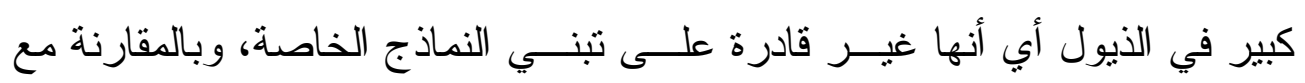

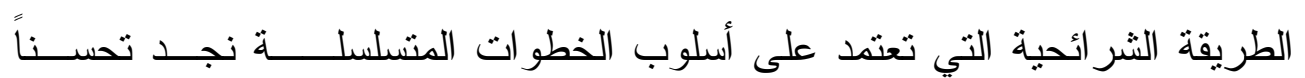

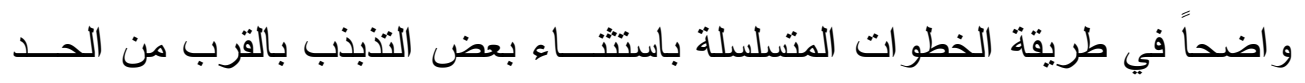

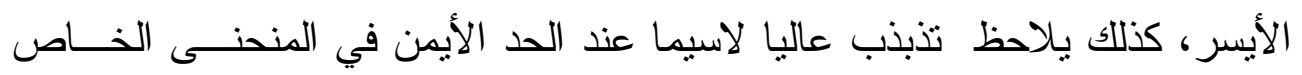

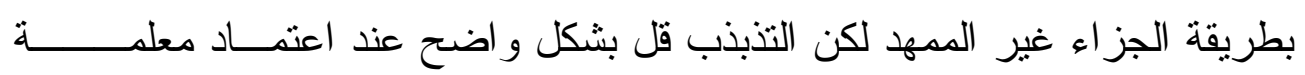

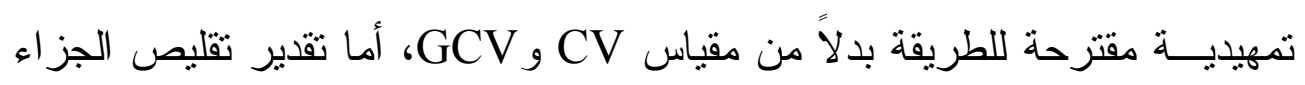

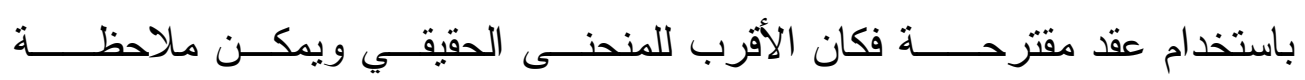

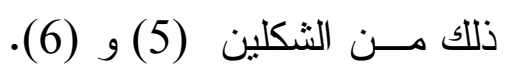
النموذج الثالث

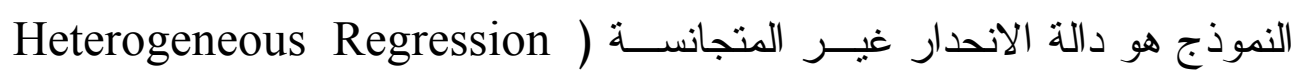
Function

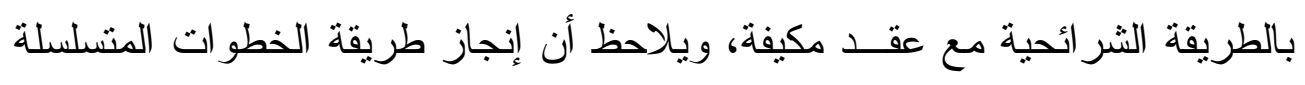
Stepwise POLYMARS الثريحة الطبيعية التكعيبية أدى إلى التخفيف من حدة التذبذب، كذلك نجد أن كــلا

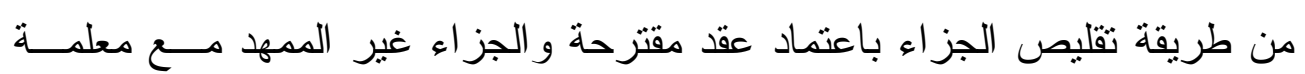

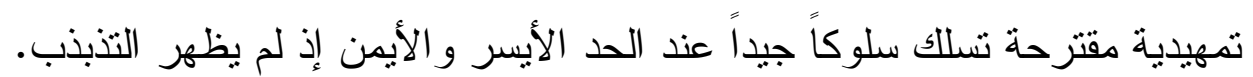

\section{4. الاستنتاجات}

في ضوء تحليل تجارب المحاكاة يمكن أدر اج اهم الاستتاجات وكما ياتي:

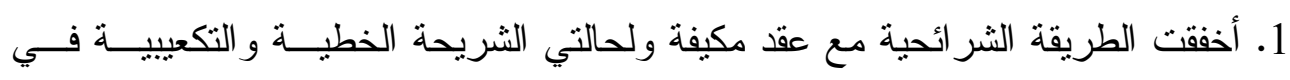

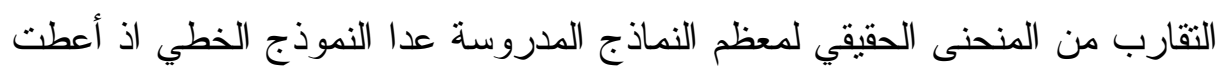
الثريحة الخطية نتائج افضل من الثريحة التكعيبية. 
2. أعطى التحوير المقترح للعقد لطريقة تقليص الجزاء نتائج افضل منها قبـلـ التحـــير

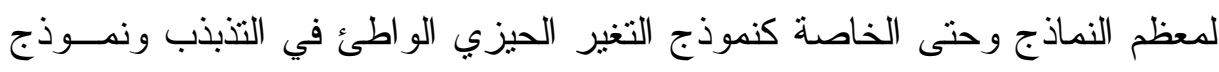

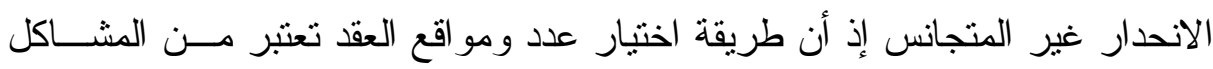

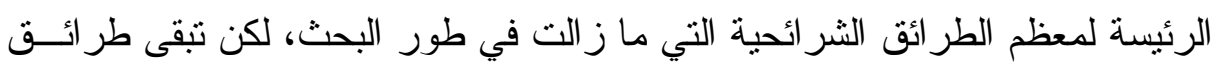

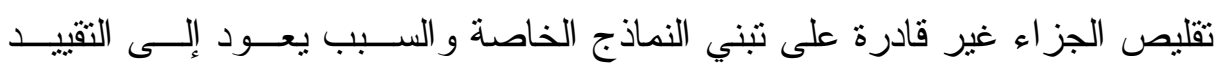
الحاصل في اختيار نموذج مرتبط بمعلمة أحادية.

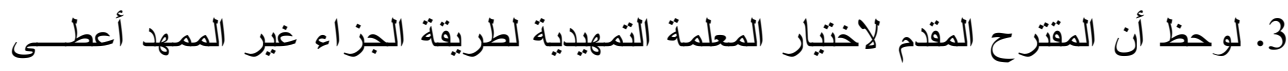

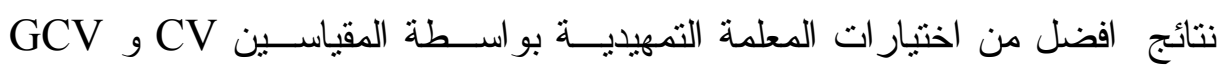
ولمعظم النماذج المدروسة. 4. أظهرت طريقة الخطو ات المنسلسلة Stepwise Stepwise POLYMARS تفورة النساً

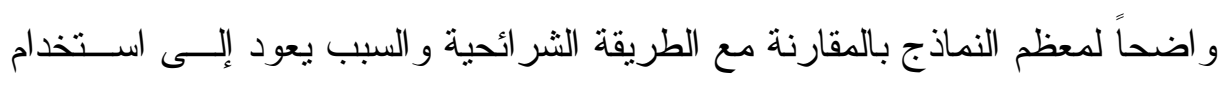

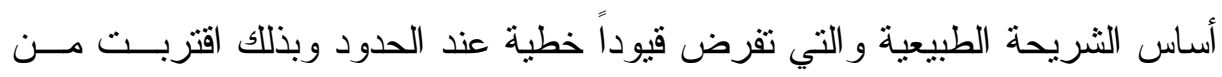

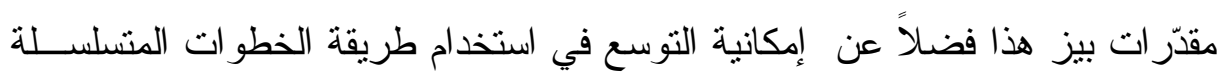
لحالات متتوعة كبيانات الاستجابة الثنائية مثلا.

5. التوصيــــــات

بناءً على ما تم التوصل إليه من استنتاجات أدناه أهم التوصيـات :

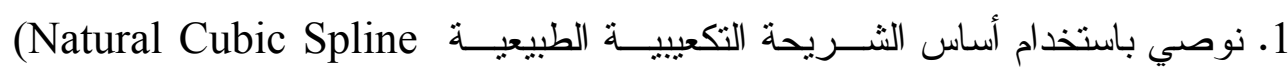

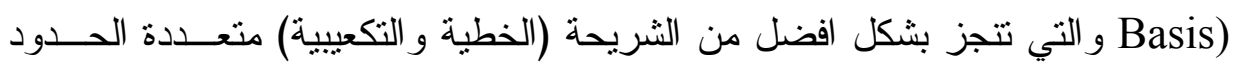
لحالة الطر ائق الثر ائحية.

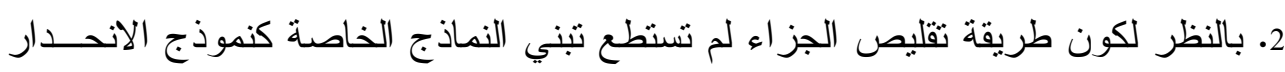

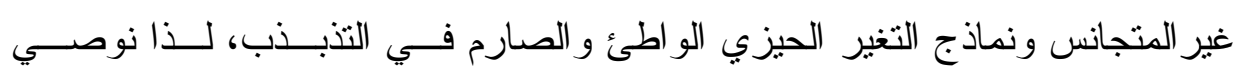

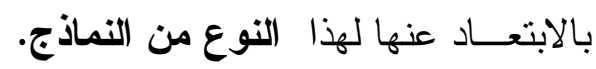

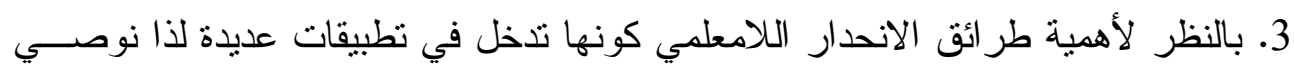

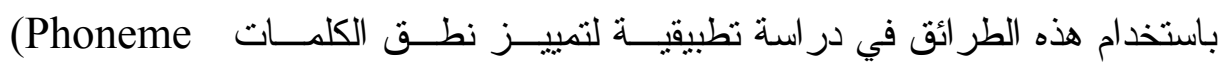
التي تربط الإحصاء كدر اسة نظرية بتطبيقات علوم الحاسبات. Recognition) 


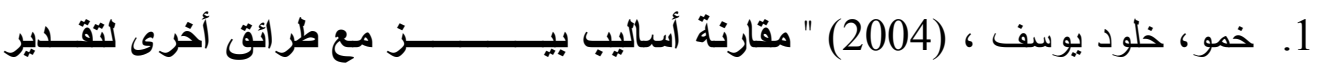

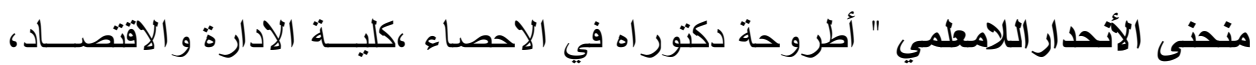

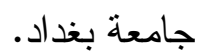

2. Friedman, J.H., (1991), "Multivariate Adaptive Regression

Splines", The Annals of Statistics, Vol. 19, 1-141.

3. Friedman, J.H. \& Silverman, B.W., (1989), "Flexible Parsimonious Smoothing and Additive Modeling", Technometrics, Vol. 31, 321.

4. Green, P.J. \& Silverman, B.W., (1994), “ Nonparametric Regression and Generalized Linear Models : A Roughness Penalty Approach", Chapman \& Hall, London.

5. Marsh, L.C. \& Cormier, D.R., (2002), "Spline Regression Models", Sage University Papers Series on Quantitative Applications in the Social Sciences Series no.07-137, Thousand Oaks, CA: Sage.

6. Stone, C.J., Hansen, M.H., Kooperberg, C. \& Truong, Y.K., (1997), "Polynomial Splines and their Tensor Products in Extended Linear Modeling", The Annals of Statistics, Vol. 25, 1371-1470.

7. Wand, M.P., (2000), “ A Comparison of Regression Spline Smoothing

Procedures", Compu. Statist., 443-462.

الجدول (1): الطرائق اللامعلمية (الطرائق الثرائحية، الجزاء غير الممهر، تقليص الجزاء، الخطوات المتسلسلة) وللنموذج الأول

\begin{tabular}{|c|l|c|c|c|c|c|c|}
\hline The & S. & \multicolumn{2}{|c|}{$\sigma=1 / 2$} & \multicolumn{2}{c|}{$\sigma=1 / 4$} & \multicolumn{2}{c|}{$\sigma=1 / 8$} \\
\cline { 3 - 8 } Method & Size & MASE & ISE & MASE & ISE & MASE & ISE \\
\hline Linear & 50 & 0.89060 & 0.44663 & 0.66447 & 0.44404 & 0.59849 & 0.43115 \\
Spline & 100 & 1.11905 & 0.52177 & 0.88133 & 0.50804 & 0.65585 & 0.50374 \\
With adap. & 200 & 1.65275 & 0.66208 & 1.64836 & 0.59614 & 1.42262 & 0.58284 \\
knot & & & & & & & \\
\hline Cubic Spline & 50 & 0.73893 & 0.31491 & 0.66656 & 0.31667 & 0.65489 & 0.31510 \\
With adap. & 100 & 1.38038 & 0.35034 & 1.19540 & 0.34529 & 0.88751 & 0.33673 \\
Knot & 200 & 2.43258 & 0.37349 & 1.65907 & 0.35701 & 0.95720 & 0.34803 \\
\hline Stepwise & 50 & 0.18077 & 0.10305 & 0.15472 & 0.10009 & 0.13154 & 0.09513 \\
POLYMAR & 100 & 0.22511 & 0.11061 & 0.19133 & 0.10025 & 0.16321 & 0.08877 \\
S & 200 & 0.27263 & 0.10027 & 0.22241 & 0.10137 & 0.20907 & 0.08455 \\
\hline Roughness & 50 & 0.12622 & 0.13679 & 0.10620 & 0.11326 & 0.10040 & 0.10704 \\
Penalty & 100 & 0.10691 & 0.11306 & 0.09676 & 0.10524 & 0.09047 & 0.10498 \\
& 200 & 0.10031 & 0.10767 & 0.09214 & 0.10251 & 0.09201 & 0.10143 \\
\hline Penalty with & 50 & 0.11432 & 0.11511 & 0.10233 & 0.10438 & 0.09154 & 0.09722 \\
Su. & 100 & 0.10422 & 0.11203 & 0.09865 & 0.10212 & 0.08351 & 0.10200 \\
Smoothing & 200 & 0.09503 & 0.10429 & 0.08424 & 0.09703 & 0.07722 & 0.09322 \\
Parameter & & & & & & & \\
\hline
\end{tabular}




\begin{tabular}{|c|l|l|l|l|l|l|l|}
\hline Penalized & 50 & 0.09499 & 0.10238 & 0.07468 & 0.08630 & 0.07127 & 0.07317 \\
Shrinkage & 100 & 0.05962 & 0.06263 & 0.04833 & 0.05177 & 0.04184 & 0.04540 \\
& 200 & 0.03382 & 0.03807 & 0.02794 & 0.02832 & 0.02413 & 0.02395 \\
\hline Penalized & 50 & 0.06739 & 0.07660 & 0.04002 & 0.05388 & 0.02948 & 0.03817 \\
Shri. with & 100 & 0.04037 & 0.04391 & 0.02575 & 0.02902 & 0.01798 & 0.02254 \\
Sugg. Knot & 200 & 0.02334 & 0.02364 & 0.01494 & 0.01545 & 0.01070 & 0.01048 \\
\hline
\end{tabular}




\begin{tabular}{|c|c|c|c|c|c|c|c|}
\hline \multirow{2}{*}{$\begin{array}{c}\text { The } \\
\text { Method }\end{array}$} & \multirow{2}{*}{$\begin{array}{l}\text { S.Si } \\
\text { ze }\end{array}$} & \multicolumn{2}{|c|}{$\sigma=1 / 2$} & \multicolumn{2}{|c|}{$\sigma=1 / 4$} & \multicolumn{2}{|c|}{$\sigma=1 / 8$} \\
\hline & & MASE & ISE & MASE & ISE & MASE & ISE \\
\hline Linear Spline & 50 & 0.96567 & 0.44061 & 0.91674 & 0.42996 & 0.74168 & 0.42655 \\
\hline With adap. & 100 & 0.93909 & 0.39885 & 0.85543 & 0.39676 & 0.69885 & 0.38907 \\
\hline Knot & 200 & 1.04954 & 0.37200 & 0.93646 & 0.38126 & 0.77634 & 0.36803 \\
\hline Cubic Spline & 50 & 0.90342 & 0.43093 & 0.85581 & 0.41313 & 0.68679 & 0.37975 \\
\hline With & 100 & 0.85127 & 0.37678 & 0.82569 & 0.36140 & 0.65338 & 0.36084 \\
\hline Adap.Knot & 200 & 0.91648 & 0.36211 & 0.87414 & 0.35346 & 0.60976 & 0.35569 \\
\hline Stepwise & 50 & 0.29491 & 0.20218 & 0.26253 & 0.18333 & 0.25516 & 0.17100 \\
\hline POLYMARS & 100 & 0.25659 & 0.18701 & 0.25617 & 0.16346 & 0.24043 & 0.14517 \\
\hline & 200 & 0.27730 & 0.16197 & 0.26482 & 0.14477 & 0.26123 & 0.14364 \\
\hline Roughness & 50 & 0.20719 & 0.25606 & 0.19261 & 0.23852 & 0.17923 & 0.22707 \\
\hline Penalty & 100 & 0.17574 & 0.21407 & 0.17323 & 0.19163 & 0.16213 & 0.17404 \\
\hline & 200 & 0.15375 & 0.19366 & 0.12623 & 0.17608 & 0.11137 & 0.15151 \\
\hline Penalty with & 50 & 0.14225 & 0.16784 & 0.13020 & 0.16277 & 0.11633 & 0.14566 \\
\hline Su. Smoothing & 100 & 0.11966 & 0.14988 & 0.11352 & 0.14201 & 0.10025 & 0.13322 \\
\hline Parameter & 200 & 0.10758 & 0.13652 & 0.09044 & 0.13192 & 0.08013 & 0.10425 \\
\hline Penalized & 50 & 0.13834 & 0.17160 & 0.12124 & 0.16624 & 0.12103 & 0.15616 \\
\hline Shrinkage & 100 & 0.11748 & 0.16958 & 0.10635 & 0.15797 & 0.10287 & 0.14214 \\
\hline & 200 & 0.11189 & 0.15167 & 0.09489 & 0.13480 & 0.09184 & 0.12190 \\
\hline Penalized & 50 & 0.04602 & 0.06141 & 0.04116 & 0.03885 & 0.03179 & 0.03676 \\
\hline Shri. with & 100 & 0.02446 & 0.02832 & 0.02235 & 0.02513 & 0.02055 & 0.02211 \\
\hline Sugg. Knot & 200 & 0.00848 & 0.02874 & 0.00812 & 0.01720 & 0.00616 & 0.00827 \\
\hline
\end{tabular}

\begin{tabular}{|c|c|c|c|c|c|c|c|}
\hline \multicolumn{7}{|l|}{ وللنموذج الثالث } & \\
\hline The Method & S.Size & MASE & ISE & MASE & ISE & MASE & ISE \\
\hline Linear Spline & 50 & 1.26922 & 0.87456 & 0.81227 & 0.72759 & 0.67768 & 0.63881 \\
\hline With adap. & 100 & 1.32908 & 0.89501 & 0.91021 & 0.75223 & 0.73358 & 0.69297 \\
\hline knot & 200 & 1.56523 & 0.92761 & 1.29901 & 0.86308 & 1.26572 & 0.82739 \\
\hline Cubic Spline & 50 & 1.07661 & 0.78513 & 0.77896 & 0.69301 & 0.64614 & 0.60639 \\
\hline With adap. & 100 & 1.14324 & 0.85369 & 0.88846 & 0.74942 & 0.84925 & 0.72174 \\
\hline Knot & 200 & 1.18385 & 0.86307 & 1.17060 & 0.84353 & 1.08889 & 0.77271 \\
\hline Stepwise & 50 & 0.19232 & 0.14070 & 0.14879 & 0.13343 & 0.13726 & 0.12375 \\
\hline POLYMARS & 100 & 0.14162 & 0.13202 & 0.13140 & 0.12152 & 0.11555 & 0.12104 \\
\hline & 200 & 0.13071 & 0.11923 & 0.11026 & 0.10325 & 0.10225 & 0.10036 \\
\hline Roughness & 50 & 0.29089 & 0.19999 & 0.27797 & 0.18895 & 0.26203 & 0.16363 \\
\hline Penalty & 100 & 0.26350 & 0.17877 & 0.25989 & 0.16580 & 0.23705 & 0.14914 \\
\hline & 200 & 0.25314 & 0.16343 & 0.21375 & 0.14443 & 0.20961 & 0.12065 \\
\hline Penalty with & 50 & 0.20632 & 0.14133 & 0.15906 & 0.14087 & 0.15598 & 0.12497 \\
\hline Su. Smoothing & 100 & 0.18386 & 0.12470 & 0.14215 & 0.11355 & 0.14019 & 0.10852 \\
\hline Parameter & 200 & 0.15032 & 0.11352 & 0.14005 & 0.10429 & 0.13221 & 0.09067 \\
\hline Penalized & 50 & 0.21989 & 0.15008 & 0.17856 & 0.14306 & 0.14523 & 0.13277 \\
\hline Shrinkage & 100 & 0.19204 & 0.13387 & 0.15773 & 0.12939 & 0.13819 & 0.12767 \\
\hline & 200 & 0.16588 & 0.12702 & 0.14305 & 0.11029 & 0.11954 & 0.10400 \\
\hline Penalized & 50 & 0.12434 & 0.11960 & 0.10598 & 0.09704 & 0.10517 & 0.08375 \\
\hline Shri. with & 100 & 0.11512 & 0.10939 & 0.09577 & 0.08018 & 0.09502 & 0.07582 \\
\hline Sugg. Knot & 200 & 0.10780 & 0.09850 & 0.07875 & 0.07417 & 0.06366 & 0.0706 \\
\hline
\end{tabular}


